Received: $\quad 2018.09 .13$

Accepted: 2018.09.26

Published: 2019.01 .16

\title{
Taxol-Resistant Gene 1 (Txr1) Mediates Oxaliplatin Resistance by Inducing Autophagy in Human Nasopharyngeal Carcinoma Cells
}

Authors' Contribution: Study Design A Data Collection B Statistical Analysis C Data Interpretation D Manuscript Preparation E Literature Search F Funds Collection G

\author{
ABCDEFG Hua-ming Chi \\ BCDEF Jing-dong Du \\ ACDE Jie Cheng \\ BCD Hua-dong Mao
}

Department of Otolaryngology, Renhe Hospital of China Three Gorges University, Yichang, Hubei, P.R. China
Corresponding Author: Source of support:

Background: Oxaliplatin (L-OHP) is an important chemotherapy regimen for nasopharyngeal carcinoma (NPC), but can fail due to drug resistance. In this study, the role of Txr1 (taxol-resistant gene 1) in oxaliplatin resistance was investigated.

Material/Methods: Cell viability assay was carried out using the CellTiter-Glo Luminescent Cell Viability Assay Kit. CNE1 and CNE2 cells were cultured continuously with gradually increasing concentrations of L-OHP for 6 months to establish drug-resistant cell lines. Autophagy was detected by electron microscopy. Txr1 expression in NPC cells was detected via Western blotting and real-time quantitative PCR (qRT-PCR).

Results: In L-OHP-resistant CNE1/L-OHP and CNE2/L-OHP cells, mRNA and protein expression of Txr1 increased compared to the parental cells, and downregulation of Txr1 re-sensitized drug-resistant cells to L-OHP. Moreover, we found that Txr1-mediated L-OHP resistance was associated with increased autophagy. Txr1-overexpression cells developed L-OHP resistance and a high level of autophagy. Inhibiting autophagy using 2 different methods - inhibition of autophagy-related gene expression and autophagy inhibitor - attenuated L-OHP resistance of NPC cells.

Conclusions: We conclude that the detection of Txr1 might become a good indicator to evaluate the treatment and prognosis of nasopharyngeal carcinoma. Our data suggest that further investigation of Txr1 in the setting of L-OHP resistance is warranted.

MeSH Keywords:

Antineoplastic Agents • Autophagy • Cell Survival

Full-text PDF: https://www.medscimonit.com/abstract/index/idArt/913180

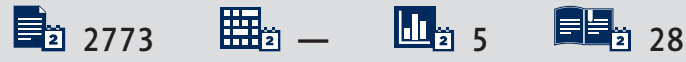




\section{Background}

Nasopharyngeal carcinoma (NPC) is one of the most prevalent malignant tumors in Southeast Asia and southern China. One of the distinctive characteristics of NPC is metastasis in the early stage [1]. The 5-year survival rate under combined treatment with adjuvant cisplatin chemotherapy and radiotherapy is $50-60 \%$, the rate of distant metastasis is $20-25 \%$, and the rate of 5-year cumulative local recurrence is $20-30 \%$ [2]. One of the main reasons behind the lethality of NPC is constant relapse and distant metastasis (3). Oxaliplatin (L-OHP), a third-generation platinum-based compound, is an important chemotherapy regimen for preventing NPC metastasis by disrupting DNA replication and transcription, but can fail due to drug resistance [4]. NPC is a complex disease, and several factors, including by environmental risk factors, EpsteinBarr virus infection, and may other genes, have been implicated in the multistep process of NPC tumorigenesis [5]. The development of drug resistance is an important factor that limits the treatment of NPC. Moreover, the molecular mechanism of drug resistance remains unclear. To develop better diagnosis and treatment methods, it is critical to identify factors related to NPC drug resistance and understand the molecular basis of drug resistance. The identification of genes contributing to chemotherapy resistance might help improving treatment of this malignancy.

Autophagy is an important process whereby cytoplasmic components, including damaged organelles and long-life proteins, after engulfment into autophagosomes, are degraded by delivery to lysosomes, constituting an intracellular regulating mechanism to maintain cellular homeostasis [6-8]. There is increasing evidence that autophagy is frequently increased in established tumors [9]. Indeed, several studies reported that advanced tumors display an "autophagy addiction" that is necessary for their energy balance $[10,11]$. It is also a crucial determinant of cancer sensitivity to chemotherapeutic drugs [12-14]. It is now clearly recognized that a critical consequence of autophagy activation in cancer cells under stress is the development of resistance to chemotherapy [13]. Several pharmacological inhibitors of autophagy have been used, and these can be categorized as early-stage or advanced-stage inhibitors of the autophagy pathway. LY294002 targeting the class III PI3K and 3-Methyladenine (3-MA) works as an early-stage inhibitor, whereas bafilomycinA1 (Baf A1) and chloroquine (CQ) are classified as advanced-stage inhibitors of autophagy by interfering with lysosomal function. The mechanism underlying regulation of the effect of autophagy on chemotherapy efficacy is unclear. Therefore, additional preclinical research is needed to understand the extent and circumstances under which blockade of autophagy can improve the therapeutic efficacy of anticancer therapies.
The mitogen-activated protein kinase (MAPK) cascades are frequently deregulated signaling pathways in human cancers (15). MAPK kinase (MEK), together with its downstream signaling extracellular signal-regulated kinase (ERK), mediate the most recently described MAPK pathway [16]. In previous studies, several types of human cancer have been described with aberrant MEK/ERK signaling [17], and cell proliferation, metastasis, and drug resistance have been reported to be associated with the MEK/ERK signaling pathway $[18,19]$. However, the full extent of molecular and cellular mechanisms by which the MEK/ ERK signaling promotes cancer pathogenesis remains unclear.

In the present study, the Taxol-resistant gene 1 (Txr1) was found to be aberrantly expressed in L-OHP resistance nasopharyngeal cancer cells. Moreover, Txr1 promoted autophagy through modulation of MEK/ERK signaling. Deficiency of autophagy induced by Txr1 can sensitize drug-resistant cells to $\mathrm{L}-\mathrm{OHP}$. These findings suggest that further investigation of Txr1 in the context of L-OHP resistance is warranted.

\section{Material and Methods}

\section{Cell culture and lentivirus infection}

NPC cell lines CNE1 and CNE2 were grown in Dulbecco's modified Eagle's medium (DMEM) (Gibco) supplemented with $10 \%$ fetal bovine serum (FBS) (Gibco). Control shRNA targeting against Lamin A (ACT GGA CTT CCA GAA GAA C) was used as a negative control. Txr1 shRNA \#1 targets against CAG TGA TAG TAG ACA AGA ATT, Txr1 shRNA \#2 targets against GGT TAG ATC ATA TAG CTA ATT. pLKO.1 plasmid was used to carry DNA oligo nucleotides loading shRNA. Green fluorescent protein-conjugated microtubule-associated protein 1 light chain 3 (GFP-LC3) was inserted into pLVX-IRES plasmids. Recombinant plasmids were co-transfected with PMD2.G and psPAX2 into HEK293T cells. The supernate medium containing lentiviruses was collected after 30-h incubation. Oxaliplatin-resistant cell lines were generated by culturing cells under gradually increased concentrations of oxaliplatin (Eloxatin, Sanofi-Aventis, France) $(0.5 \mu \mathrm{g} / \mathrm{mL}$ to $8 \mu \mathrm{g} / \mathrm{mL})$ over a 6 -month period.

\section{Real-time quantitative PCR (qRT-PCR) analysis}

Total RNA was extracted from $5 \times 10^{6}$ cells using TRIzol reagent (Invitrogen), and subjected to reverse transcription PCR for detection of Txr1 and ACTB mRNA levels. The 7900 Real-Time PCR (Applied Biosystems) was used for real-time quantitative PCR. Three assays were carried out for each reaction tube. Data were analyzed with the $2^{-\Delta \Delta C t}$ method using ACTB as an internal control. The cycling conditions were $95^{\circ} \mathrm{C}$ for $20 \mathrm{~s}$, followed by 40 cycles of $95^{\circ} \mathrm{C}$ for $15 \mathrm{~s}$, and $58^{\circ} \mathrm{C}$ for $1 \mathrm{~min}$. All primers were designed online according to the previous literature [20]. 


\section{Cell viability assay}

Cell viability assay was carried out using the CellTiter-Glo Luminescent Cell Viability Assay Kit (G7571, Promega) following the manufacturer's protocol. A graph was plotted using GraphPad Prism5 software.

\section{Western blotting analysis}

NPC cell lysates were prepared using lysis buffer $(25 \mathrm{mM}$ Tris$\mathrm{HCl} \mathrm{pH}=7.4,0.1 \%$ SDS, $150 \mathrm{mM} \mathrm{NaCl}, 1 \mathrm{mM}$ EDTA, 10\% glycerol, $5 \mathrm{mM} \mathrm{MgCl}, 2 \mathrm{mM} \mathrm{Na}_{3} \mathrm{VO}_{4}, 0.1 \mathrm{mM}$ EGTA, 1\% NP-40, $1 \mathrm{mM}$ PMSF (CST \#8553), and $1 \times$ Cocktail (CST \#5871)) on ice for $25 \mathrm{~min}$. Cell lysates were centrifuged at $12000 \mathrm{rpm}$ at $4^{\circ} \mathrm{C}$ for $10 \mathrm{~min}$. The supernatants were gathered into new EP tubes and the total protein concentration was quantified by BCA method, and then denatured at $100^{\circ} \mathrm{C}$ for $5 \mathrm{~min}$ and cooled on ice. Western blotting was performed following standard procedures [21]. Primary antibodies used were $\beta$-actin (Sigma, A1978), LC3 (Cell Signaling Technologies (CST), \#2775), Atg7 (CST, \#2631), Atg5 (CST, \#2630), and Txr1 (LifeSpan Biosciences, LS-C116668). Secondary antibodies were purchased from ZSGB Biotechnology (Beijing, China).

\section{Fluorescence microscopy}

NPC cells stably expressing pEGFP-LC3 were grown on 6-cm culture dishes. A fluorescence microscope (Leica) was used to observe cells expressing GFP-LC3. Cell images were obtained with a CCD camera (Coolsnap) with METAMORPH software (MDS Analytical Technologies, Canada). At least 100 cells from 10 different fields were counted for statistical analysis, and significance of differences was analyzed by use of the $t$ test.

\section{Transmission electron microscopy (TEM)}

For electron microscopy, the cells were fixed in a solution of $4 \%$ glutaraldehyde $0.05 \mathrm{M}$ sodium cacodylate, postfixed in $1.5 \%$ $\mathrm{OsO}_{4}$, and dehydrated in alcohol. They were then prepared for flat embedding in Epon 812 and then observed using a Zeiss CEM 902 electron microscope.

\section{Statistical analysis}

We used one-way ANOVA followed by Tukey's test using GraphPad Prism 5.0 software for data analysis. Statistical significance was calculated using data from at least 3 independent experiments. Data are presented as the mean \pm standard deviation (SD). Differences were considered statistically significant at $\mathrm{P}<0.05$.

\section{Results}

\section{Oxaliplatin treatment induces the expression of Txr1 in human nasopharyngeal cancer cells CNE1 and CNE2}

Taxol-resistant gene 1 (Txr1) is a drug-resistant gene found by Cohen's team [22]. It has been confirmed that Txr1 is expressed differently in nasopharyngeal carcinoma, non-small cell lung cancer (NSCLC), gastric cancer (GC), and breast cancer, in which Txr1 mRNA expression detection in fresh tumor tissue was deemed to an independent prognostic factor $[20,23,24]$. To explore the role of Txr1 in oxaliplatin (L-OHP) treatment of nasopharyngeal cancer cell, we cultured CNE1 and CNE2 cells in medium blending L-OHP. The levels of Txr1 mRNA and protein were detected at different timepoints (Figure $1 \mathrm{~A}$ ) and at different doses of L-OHP (Figure 1B), showing that TSP1 is the downstream suppressant gene of Txr1. To explore the expression of Txr1 in drug-resistant nasopharyngeal cancer cells, we performed real-time quantitative PCR (qRT-PCR) analysis to examine Txr1 gene transcription (Figure 1C). Western blotting was carried out to detect protein levels of Txr1 in CNE1/L-OHP, CNE2/L-OHP, and the parental cells (Figure 1C). The data indicated that CNE1/L-OHP and CNE2/L-OHP cells expressed higher levels of Txr1 compared to the parental cells. To further confirm whether increased Txr1 promotes L-OHP resistance of nasopharyngeal cancer cells, Txr1 was overexpressed in CNE1 and CNE2 cells using lentivirus. Then, cell viability analysis was carried out in the condition of L-OHP treatment (Figure 1D). The results clearly showed that overexpression of Txr1 increased resistance to L-OHP treatment in CNE1 and CNE2 cells $(P<0.01)$.

\section{Autophagy induced by oxaliplatin protects CNE1 and CNE2 cells from the cytotoxicity of oxaliplatin}

Autophagy is an important mechanism of cellular homeostasis in response to stress. To determine whether autophagy is involved in L-OHP treatment in CNE1 and CNE2 cells, the microtubule-associated protein light-chain3 (LC3) and Atg5 were examined using Western blotting assay in the condition of L-OHP treatment (Figure 2A, 2B). Significantly higher LC3II/LC3I and Atg5 levels were observed in a time- and dose-dependent manner. Therefore, we hypothesized that autophagy is a mechanism underlying L-OHP resistance in CNE1 and CNE2 cells. To determine the state of cellular autophagy in CNE1/L-OHP, CNE2/L-OHP, and parental cells, pEGFP-LC3 plasmid was transfected into cells and GFP-LC3 puncta were observed through a fluorescence microscope, with serum starvation treatment as a positive control (Figure 2C). The cell images demonstrated that the average number of GFP-LC3 puncta in L-OHP-resistant cells was higher than in parental cells $(P<0.05)$. Transmission electron microscopy (TEM) images indicated that there was more autophagosomes formation in L-OHP-resistant cells than in parental cells (Figure 2D). To further characterize the 

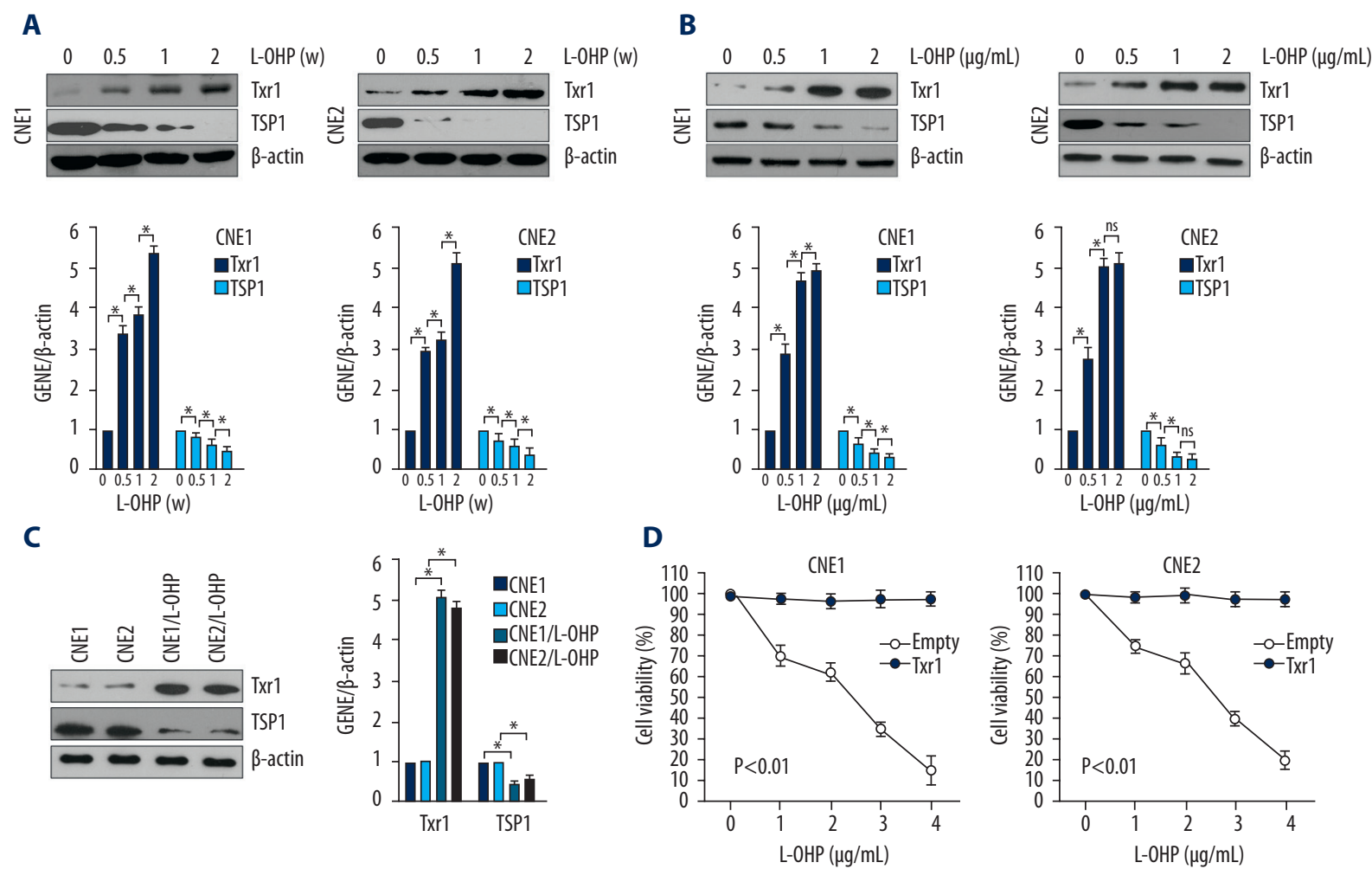

Figure 1. L-OHP induced the expression of Txr1 in CNE1 and CNE2 cells. (A) CNE1 and CNE2 cells were treated with L-OHP (1 $\mu \mathrm{g} / \mathrm{mL})$ for $0,0.5,1$, or 2 weeks. Total RNA and cell lysates were prepared and subjected to qRT-PCR and Western blotting analysis. $\beta$-actin was used as a loading control. (B) CNE1 and CNE2 cells were treated with the indicated concentrations of L-OHP for $24 \mathrm{~h}$. Total RNA and cell lysates were prepared and subjected to qRT-PCR and Western blotting analysis. $\beta$-actin was used as a loading control. (C) Lysates of acquired L-OHP-resistant cells and parental cells were examined using indicated antibodies (left), and mRNA levels were examined using qRT-PCR (right). (D) Cell viability assay was carried out in cells overexpressing Txr1 and in control cells, with or without L-OHP treatment $(n=3)$. Data are mean \pm SEM of 3 independent replicates, * $P<0.05$.

effects of autophagy in L-OHP resistance, autophagy was inhibited using bafilomycin A1 (Baf A1) or shRNA against Atg5. The deficiency of autophagy impaired the L-OHP resistance in CNE1/L-OHP cells ( $<<0.01$, Figure $2 \mathrm{E}$ ). When CNE2/L-OHP cells were detected in this study, similar results were observed (data not shown). These results indicate that autophagy is induced by L-OHP treatment and protects cells from the cytotoxicity of L-OHP.

\section{Heightening of Txr1 promotes autophagy and oxaliplatin resistance in CNE1 and CNE2 cells.}

Our data indicate that overexpression of Txr1 increased resistance to L-OHP in CNE1 and CNE2 cells (Figure 1D) and that autophagy contributed to L-OHP resistance in CNE1 and CNE2 cells (Figure 2E). To determine if Txr1 affects cellular autophagy, CNE1 and CNE2 cells were stably overexpressed Txr1 by lentivirus system. As shown in Figure 3A, autophagy markers were significantly increased after Txr1 overexpression. When Txr1 was overexpressed in cells transfected with pEGFP-LC3, GFPLC3 puncta were obviously increased compared to control cells $(P<0.05$, Figure $3 B)$. These results confirmed that Txr1 can promote autophagy in CNE1 and CNE2 cells. However, whether the regulation of L-OHP resistance by Txr1 was through autophagy remains unclear. To discover the link between autophagy induced by Txr1 and L-OHP resistance, Txr1-overexpression cells were treated with Baf A1 or shRNA against Atg5 to impair autophagy, then cell viability was evaluated after L-OHP treatment (Figure 3C, 3D). The data showed that Txr1 promoted $\mathrm{L}-\mathrm{OHP}$ resistance in CNE1 and CNE2 cells, and blockade of autophagy decreased L-OHP resistance induced by Txr1 $(P<0.01)$.

\section{Loss of Txr1 precludes CNE1/L-OHP and CNE2/L-OHP cells from tolerating oxaliplatin.}

To further characterize the function of Txr1 on autophagy, CNE1/L-OHP and CNE2/L-OHP cells expressing high levels of Txr1 were incubated with lentivirus carrying Txr1 shRNA for 
A

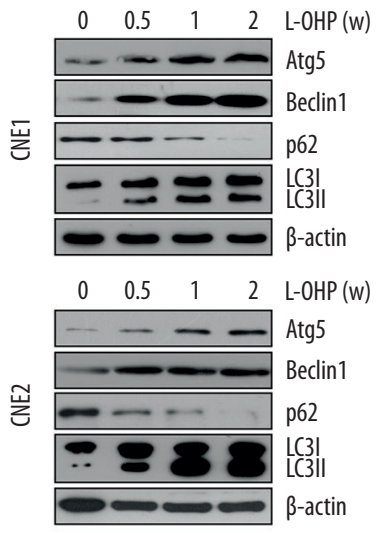

B

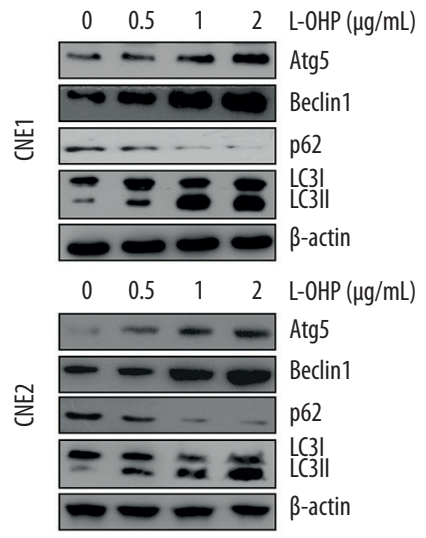

C
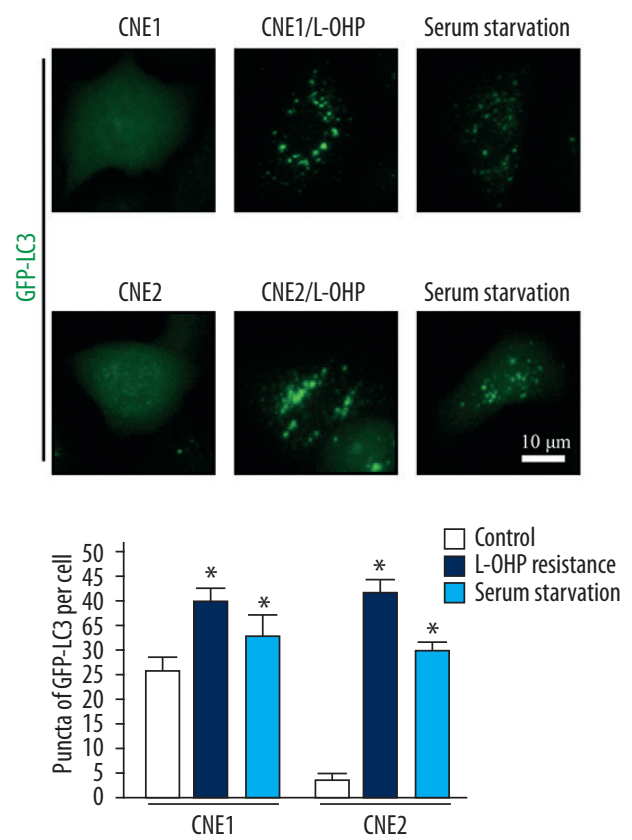

CNE1

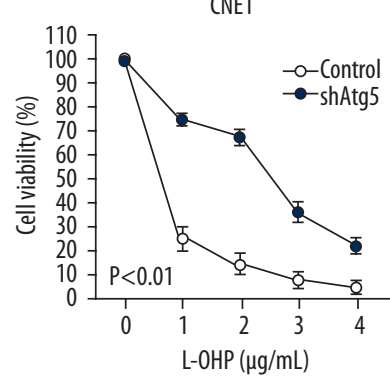

CNE2

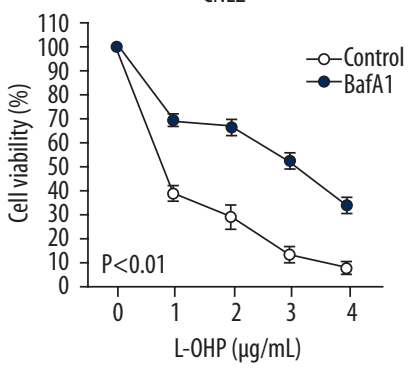

Figure 2. Autophagy induced by L-OHP protects CNE1 and CNE2 cells from the cytotoxicity of L-OHP. (A) CNE1 and CNE2 were treated for the indicated times with of L-OHP $(1 \mu \mathrm{g} / \mathrm{mL})$. Cell lysates were prepared and subjected to Western blotting analysis. $\beta$-actin was used as a loading control. (B) CNE1 and CNE2 cells were treated with the indicated concentrations of L-OHP for $24 \mathrm{~h}$. Cell lysates were prepared and subjected to Western blotting analysis. $\beta$-actin was used as a loading control. (C) Representative fluorescence microscope images of drug-resistant cells and parental cells stably expressing GFP-LC3, with serum starvation as a positive control. Scale bar: $10 \mu \mathrm{m}$. (D) Transmission electron microscopy (TEM) images of drugresistant cells and parental cells. Scale bar: $5 \mu \mathrm{m}$, ' $A$ ' indicates autophagosome. (E) CNE1 and CNE2 cells were inhibited autophagy by Baf A1 $(10 \mathrm{nM})$ or transfected with shRNA targeting Atg 5 or control shRNA, followed by treatment with indicated concentrations of L-OHP or vehicle for $24 \mathrm{~h}$. Cell viability was measured using the Cell Viability Assay Kit. Results shown are representative of 3 independent experiments, ${ }^{*} \mathrm{P}<0.05$.

knockdown of Txr1. As expected, autophagy was significantly depressed after loss of Txr1 in CNE1/L-OHP and CNE2/L-OHP cells (Figure 4A). Yet, more than any these, the ability of tolerating L-OHP was obviously suppressed after knockdown of Txr1 in CNE1/L-OHP and CNE2/L-OHP cells ( $P<0.01$, Figure 4B, 4C). These data indicate that Txr1 was requisite for toleration of L-OHP in NPC cells.

\section{Txr1 modulates oxaliplatin resistance and autophagy through MEK/ERK signaling in CNE1 and CNE2 cells}

As previously reported, the extracellular signal-regulated kinase (ERK) cascade can be activated by a variety of stimuli, and it plays an important role in the regulation of cell differentiation, autophagy, and survival [25], and mitogen-activated protein kinase (MEK) was verified to act as an upstream signal of ERK. However, whether the MEK/ERK signaling pathway plays a role in autophagy induced by Txr1 in L-OHP-resistant 
A

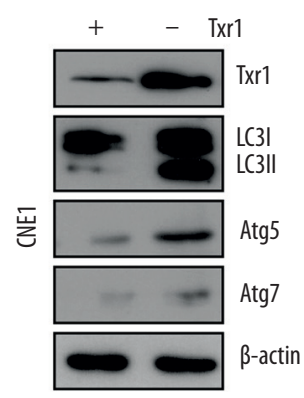

B

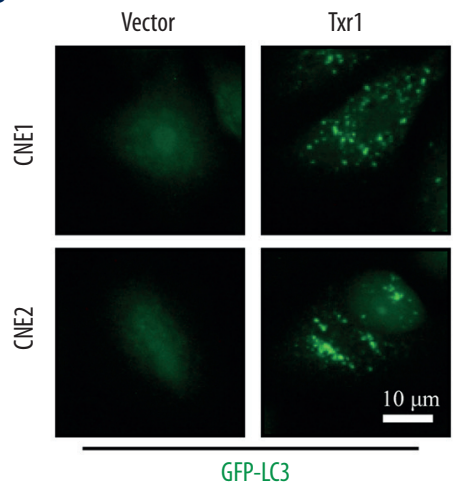

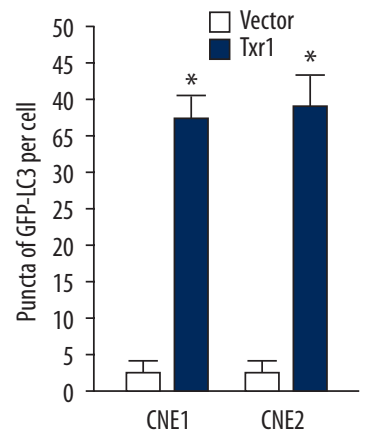

CNE1

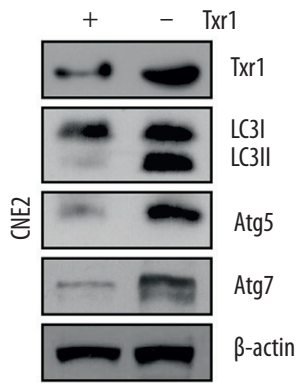

D

C
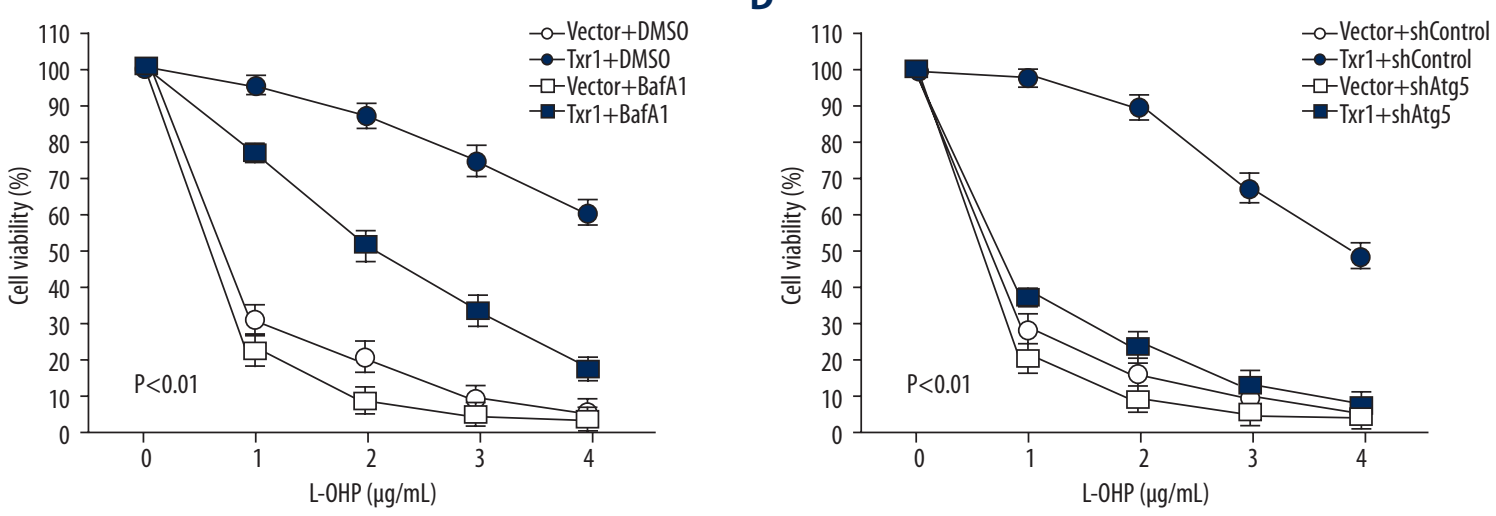

Figure 3. Txr1 promotes autophagy and oxaliplatin resistance in CNE1 and CNE2 cells. (A) Autophagy-related genes were examined by Western blotting assay after overexpression of Txr1 gene in CNE1 and CNE2 cells. (B) Representative fluorescence microscope images of Txr1-overexpression cells stably expressing GFP-LC3, with serum starvation as a positive control. Scale bar: $10 \mu \mathrm{m}$. (C, D) Txr1-overexpression cells were inhibited autophagy by Baf A1 (10 nM) or transfected with shRNA targeting Atg5 or control shRNA, followed by treatment with indicated concentrations of L-OHP or vehicle for $24 \mathrm{~h}$. Cell viability was measured by use of the Cell Viability Assay Kit. Results shown are representative of 3 independent experiments, * $P<0.05$.

NPC cells has been unclear. In the present study, we tried to determine the function of the MEK/ERK signaling pathway in Txr1-induced chemotherapy and autophagy in NPC cells. Txr1 gene was overexpressed in CNE1 and CNE2 cells, and then MEK1/2 and PERK1/2 were examined via Western blotting assay (Figure 5A). The results clearly indicated that MEK/ ERK signaling was activated by Txr1. We found that Txr1 promotes autophagy in CNE1 and CNE2 cells (Figure 3A, 3B). To further determine the role of the MEK/ERK pathway in autophagy induced by Txr1, we treated cells expressing Txr1 with the pERK-specific inhibitor GSK2656157 (1 $\mu \mathrm{M})$ [26]. We found that Txr1-induced autophagy was significantly decreased when cells were exposed to GSK2656157 (Figure 5B, 5C), and, similarly, MEK inhibitor GSK1120212 exposure remarkably impaired active PERK and autophagy induced by Txr1. This indicates a key role of MEK/ERK signaling in regulation of Txr1induced autophagy. In the next experiment, we found that GSK2656157 could re-sensitize drug-resistant cells to L-OHP
$(P<0.05$, Figure 5D). Taken together, our results show thatTxr1 modulates L-OHP resistance and autophagy through MEK/ERK signaling in CNE1 and CNE2 cells.

\section{Discussion}

Nasopharyngeal carcinoma (NPC) is the most common head and neck malignancy in Southeast Asia and southern China. One of the distinctive characteristics of NPC is metastasis in the early stage [1]. Given its high sensitivity to radiotherapy, the most common treatment for NPC is radiotherapy. However, radioresistance and the damage of radiation to normal tissues are major obstacles to successful treatment. Distant metastasis often occurs after therapy, which is one of the causes for NPC-related death in some patients, although NPC is confirmed to be sensitive to radiotherapy [3]. Thus, special emphasis is needed on the development of more effective chemotherapeutic agents. 
A
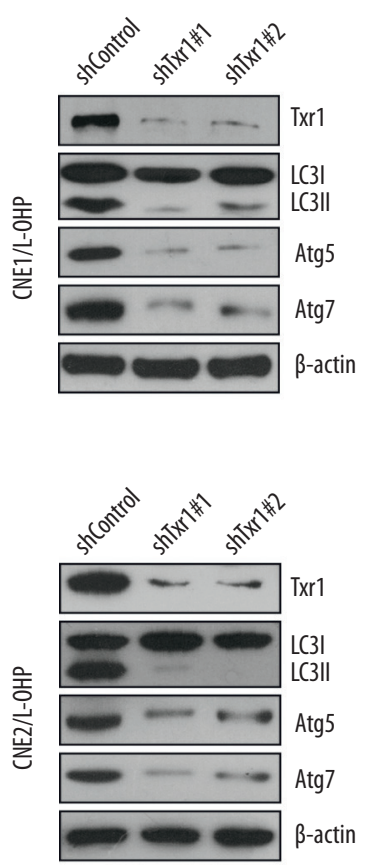

B
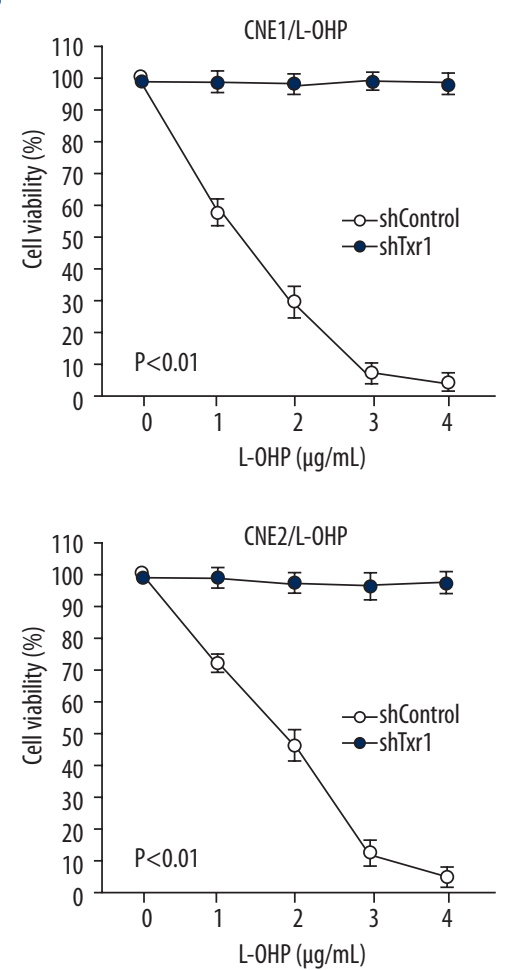

C
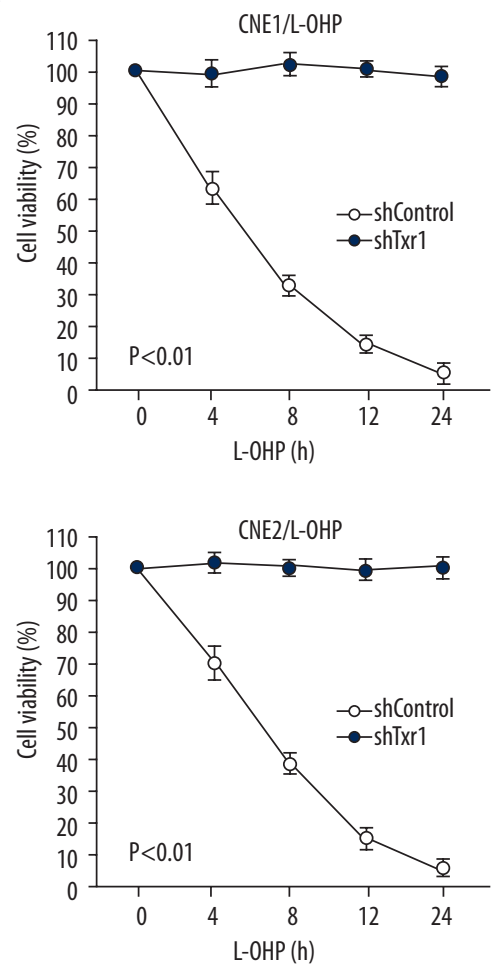

Figure 4. Loss of Txr1 precludes CNE1/L-OHP and CNE2/L-OHP cells from tolerating L-OHP. (A) Autophagy-related genes were examined by Western blotting assay after knockdown of the Txr1 gene in CNE1/L-OHP and CNE2/L-OHP cells. (B) Drugresistant cells were transfected with shRNA targeting Txr1 or control shRNA, followed by treatment with indicated concentrations of L-OHP or vehicle for $24 \mathrm{~h}$. Cell viability was detected by use of the Cell Viability Assay Kit. (C) Drugresistant cells were transfected with shRNA targeting Txr1 or control shRNA, followed by treatment for indicated times with L-OHP $(5 \mu \mathrm{g} / \mathrm{mL})$. Cell viability assay was carried. Results shown are representative of 3 independent experiments.

Oxaliplatin (L-OHP) is a third-generation platinum-based compound commonly used to treat metastatic malignant cancers. L-OHP contributes to tumor therapy by interrupting DNA replication and transcription processes through binding to DNA and generating platinum-DNA adducts [4]. Unfortunately, resistance to chemotherapy may cause failure of cell death activated in response to drug treatment. Here, we have shown that Txr1 is induced by treatment with L-OHP in a time- and dose-dependent manner in CNE1 and CNE2 cells, and overexpression of Txr1 plays an important role in L-OHP resistance in NPC cells. These findings suggest that Txr1 might be a major contributor to the L-OHP resistance of NPC, and Txr1 expression could be used as a biomarker for predicting treatment outcomes of individual with NPC treated with L-OHP. In previous studies, Txr1 was reported to affect the efficacy of L-OHP chemotherapy through reducing apoptosis mediated by thrombospondin-1 (TSP-1), and Txr1 is also reported to be associated with poor prognosis in patients with gastric cancer [20].
In this study, we revealed that L-OHP resistance NPC cells showed higher levels of Txr1 expression and autophagy than that in parental cells, and loss of Txr1 obviously suppressed autophagy in L-OHP-resistant NPC cells. Inhibition of autophagy using different methods impaired the resistance to L-OHP in drug-resistant NPC cells. These findings suggest that autophagy activated by L-OHP is a protective factor for CNE1 and CNE2 cells tolerating the cytotoxicity of L-OHP. A variety of data have demonstrated that autophagy is critically involved in drug resistance of cancer. In Zhang's study, autophagy activated by CD44v6 contributed to acquired drug resistance by the MAPKRas-ERK pathway in colorectal cancer [21]. McKenna et al. revealed that induction of compromised autophagy contributed to promotion of cytotoxicity of chemotherapeutic agents [22], and oxaliplatin resistance was apparently recovered through blockade of autophagy in hepatocellular carcinoma cells [23]. These findings point to the potential of treating cancer by perturbing autophagy. 


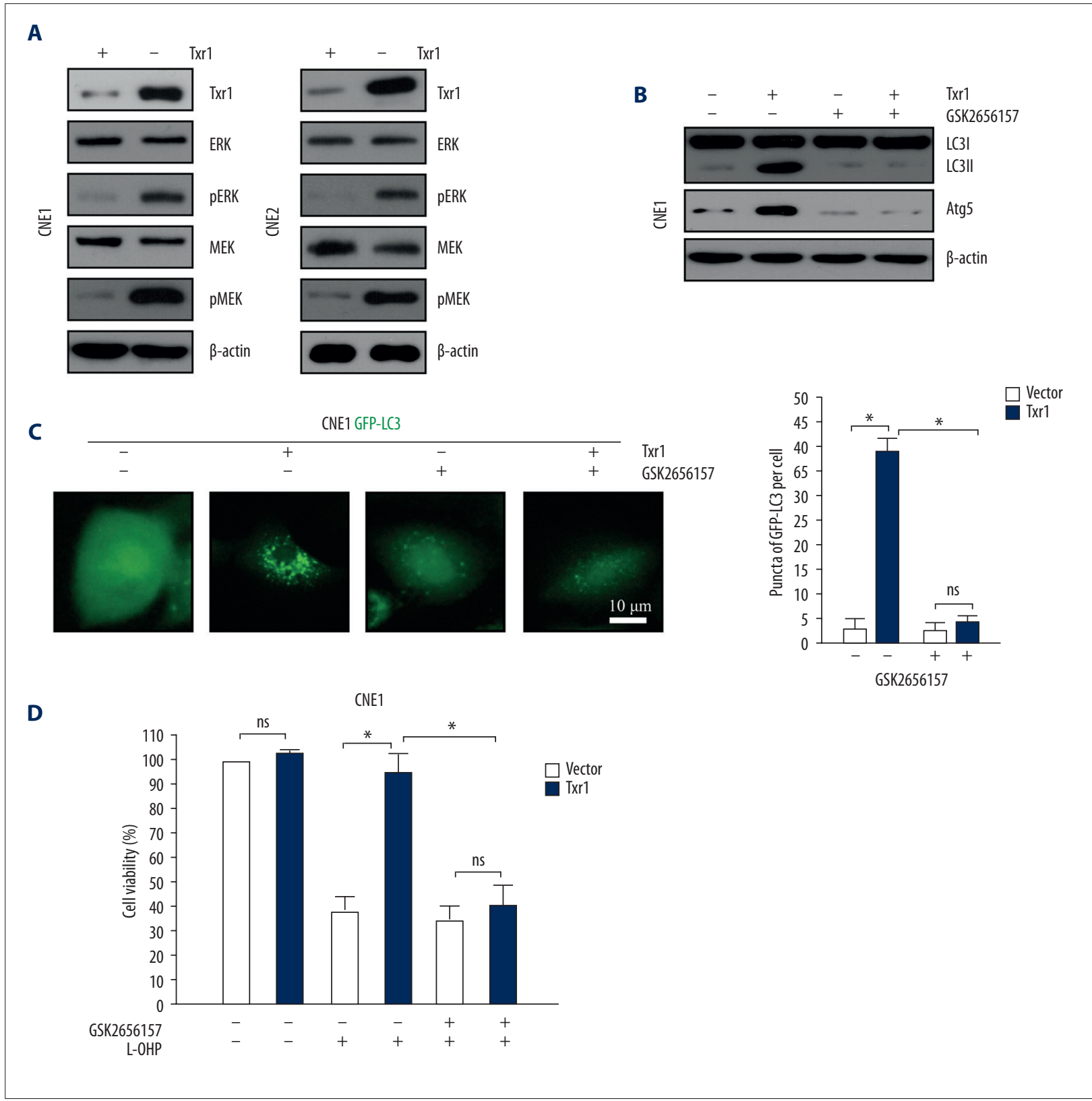

Figure 5. Txr1 modulates L-OHP resistance and autophagy through MEK/ERK signaling in CNE1 and CNE2 cells. (A) MEK/ERK signaling was examined by Western blotting assay after overexpression of Txr1 gene in CNE1 cells. (B) Autophagy-related genes were examined in Txr1-overexpression cells treated with GSK2656157 (1 $\mu$ M). (C) Txr1 overexpression cells stably expressing GFP-LC3 were treated with GSK2656157 (1 $\mu \mathrm{M})$ for $24 \mathrm{~h}$. Representative fluorescence microscope images are shown, with serum starvation as a positive control. Scale bar: $10 \mu \mathrm{m}$. (D) Txr1-overexpression cells were exposed to MEK/ERK inhibitor GSK2656157 $(1 \mu \mathrm{M})$ for $6 \mathrm{~h}$, then treated cells with GSK2656157 $(1 \mu \mathrm{M})$ and L-OHP for another $18 \mathrm{~h}$. Cell viability assay was carried out in time. Results shown are representative of 3 independent experiments, ${ }^{*} \mathrm{P}<0.05$.

The MEK/ERK signaling pathway has important roles in human cancer cells [24]. Regulation of this signaling pathway is critical for cell survival and induction of cell death in cancer cells. In the present study, we found that Txr1 contributed to resistance to L-OHP by modulating autophagy through the MEK/ ERK signaling pathway in NPC cells. A previous study reported that ursodeoxycholic acid-induced cell death was observed in gastric cancer cells, suggesting the involvement of MEK/ERK activation in regulation of pro-apoptosis [24], and MEK/ERK signaling activation was also shown to arrest cell cycle at $S$ phase in the context of hydroxyl urea [25]. However, the MEK/ERK pathway also promoted OA1-induced melanoma cell 
migration [26]. Apoptosis mediated by DHA was accompanied by inactivation of the MEK/ERK signaling pathway in glioma cells [27]. Obviously, the role of MEK/ERK in human cancers is complex. Our data indicate that inactivation of the MEK/ERK pathway decreased NPC cells growth upon exposure to L-OHP.

\section{References:}

1. Adham M, Kurniawan AN, Muhtadi Al et al: Nasopharyngeal carcinoma in Indonesia: Epidemiology, incidence, signs, and symptoms at presentation. Chin J Cancer, 2015; 31: 185-96

2. Siddique MA, Sabur MA, Kundu SC et al: Difficulty in diagnosis of nasopharyngeal carcinoma. Mymensingh Med J, 2012, 21: 158-61

3. Chang ET, Adami HO: The enigmatic epidemiology of nasopharyngeal carcinoma. Cancer Epidemiol Biomarkers Prev, 2016; 15: 1765-77

4. Saif MW, Choma A, Salamone SJ et al: Pharmacokinetically guided dose adjustment of 5-fluorouracil: A rational approach to improving therapeutic outcomes. J Natl Cancer Inst, 2015; 101: 1543-52

5. Chou J, Lin YC, Kim J et al: Nasopharyngeal carcinoma - review of the molecular mechanisms of tumorigenesis. Head Neck, 2008; 30: 946-63

6. Barth S, Glick D, Macleod KF: Autophagy: assays and artifacts. J Pathol, 2010; 221: 117-24

7. Klionsky DJ, Abdelmohsen K, Abe A et al: Guidelines for the use and interpretation of assays for monitoring autophagy (3rd edition). Autophagy, 2016; 12: 1-222

8. Mizushima N, Yoshimori T, Levine B: Methods in mammalian autophagy research. Cell, 2010; 140: 313-26

9. Chen S, Rehman SK, Zhang W et al: Autophagy is a therapeutic target in anticancer drug resistance. Biochim Biophys Acta, 2010; 1806: 220-29

10. Lum JJ, Bauer DE, Kong $M$ et al. Growth factor regulation of autophagy and cell survival in the absence of apoptosis. Cell, 2015; 120: 237-48

11. White E: Deconvoluting the context-dependent role for autophagy in cancer. Nat Rev Cancer, 2012; 12: 401-10

12. Duffy A, Le J, Sausville E et al: Autophagy modulation: A target for cancer treatment development. Cancer Chemother Pharmacol, 2015; 75: 439-47

13. Sui $X$, Chen $R$, Wang $Z$ et al: Autophagy and chemotherapy resistance: $A$ promising therapeutic target for cancer treatment. Cell Death Dis, 2013; 2: 5-11

14. Wu WK, Coffelt SB, Cho $\mathrm{CH}$ et al: The autophagic paradox in cancer therapy. Oncogene, 2012; 31: 939-53

15. Feldt $M$, Bjarnadottir $O$, Kimbung $S$ et al: Statin-induced anti-proliferative effects via cyclin D1 and p27 in a window-of-opportunity breast cancer trial. J Transl Med, 2015; 13: 133-38

\section{Conclusions}

The present preliminary in vitro exploration of the mechanisms and associations between Txr1 expression and L-OHP resistance of NPC cells should be of value in developing further experiments. Our data suggest that Txr1 is a potential therapeutic target for L-OHP resistance NPC.

16. Bjarnadottir O, Romero Q, Bendahl PO et al: Targeting HMG-CoA reductase with statins in a window-of-opportunity breast cancer trial. Breast Cancer Res Treat, 2013; 138: 499-508

17. Hindler K, Cleeland CS, Rivera $\mathrm{E}$ et al: The role of statins in cancer therapy. Oncologist, 2006; 11: 306-15

18. Chan KK, Oza AM, Siu LL: The statins as anticancer agents. Clin Cancer Res, 2003; 9: 10-19

19. Liu W, Zhang Z, Zhang Y et al: HMGB1-mediated autophagy modulates sensitivity of colorectal cancer cells to oxaliplatin via MEK/ERK signaling pathway. Cancer Biol Ther, 2015; 16: 511-17

20. Bai ZG, Qu X, Han W et al: Expression of taxol resistance gene 1 correlates with gastric cancer patient clinical outcome and induces taxol resistance. Mol Med Rep, 2010; 3: 1071-78

21. Lv L, Liu HG, Dong SY et al: Upregulation of CD44v6 contributes to acquired chemoresistance via the modulation of autophagy in colon cancer SW480 cells. Tumour Biol, 2016; 3: 5-9

22. O'Donovan TR, Rajendran S, O'Reilly S et al: Lithium modulates autophagy in esophageal and colorectal cancer cells and enhances the efficacy of therapeutic agents in vitro and in vivo. PLoS One, 2015; 10: e0134676

23. Du H, Yang W, Chen L et al: Role of autophagy in resistance to oxaliplatin in hepatocellular carcinoma cells. Oncol Rep, 2012; 27: 143-50

24. Shaw RJ, Cantley LC: Ras, PI(3)K and mTOR signalling controls tumour cell growth. Nature, 2006; 441: 424-30

25. Lim SC, Duong HQ, Parajuli KR et al: Pro-apoptotic role of the MEK/ERK pathway in ursodeoxycholic acid-induced apoptosis in SNU601 gastric cancer cells. Oncol Rep, 2012; 28: 1429-34

26. Wu D, Chen B, Parihar $\mathrm{K}$ et al: ERK activity facilitates activation of the S-phase DNA damage checkpoint by modulating ATR function. Oncogene, 2006; 25: 1153-64

27. Bai J, Xie X, Lei $Y$ et al: Ocular albinism type 1-induced melanoma cell migration is mediated through the RAS/RAF/MEK/ERK signaling pathway. Mo Med Rep, 2014; 10: 491-95

28. Du W, Pang C, Xue $Y$ et al: Dihydroartemisinin inhibits the Raf/ERK/MEK and PI3K/AKT pathways in glioma cells. Oncol Lett, 2015; 10: 3266-70 